\title{
Graphical Stabilization Approach for Unstable First Order Linear Systems with Time Delay
}

\author{
A. Maddi*1, A. Guessoum ${ }^{2}$, D. Berkani ${ }^{3}$
}

Accepted : 12/05/2017 Published: 21/08/2017

DOI: 10.18100/ijamec.2017Special Issue30464

Abstract: In this paper, a graphical stabilization approach is proposed and analyzed for a class of unstable first order linear systems with time delay. We first show that the control designs based on time invariant models are unable to guarantee stability and asymptotic tracking for unstable first order linear systems in general case. So, the condition stability is analysed graphically by computing the first derivative and plotting the graph of a function with precision; the first derivative allows us to determine the critical points and several conditions of stability. Therefore, it's important to note that the method can guarantee the existence of a proportional gain to ensure the stability of the closed-loop system such that the time delay is small relatively to the time constant. Finally, a numerical example illustrates the efficiency and performances of the proposed approach.

\section{Keywords: asymptotic stability, linear system, output feedback, proportional gain, time delay}

\section{Introduction}

The time delay phenomena were first appeared in biological phenomena and were later found in many engineering systems, such as mechanical transmissions, fluid transmissions, and networked control systems. They are considered as a source of instability and poor control performance. The main time-domain methods are the Lyapunov-Krasovskii functional and Razumikhin function methods $[1,2]$. They are the most common approaches to the stability analysis of time-delay systems. Since it was very difficult to construct Lyapunov-Krasovskii functional and Lyapunov functions until 1990, the stability criteria obtained were generally in the form of existence conditions and do not have a general solution. For example, a new LyapunovKrasovskii functional is introduced to take into account that a nominal delay may be different from zero. Also, as it has been shown in $[3,4,5,6]$ and references therein, there exist systems that are unstable in the case free of delays and becomes stable only for some strictly positive values of the delay. In [7], the observer based approach is considered for stabilization and control of unstable first-order plants with known parameters. An alternative way to prove the stability of time-delay systems using Taylor series and the quadratic separation formalism is exposed in [8]. Another is concerned with the stability analysis for uncertain systems with interval time-varying delays based on the division of the time delay range [9].

This paper addresses this problem of proving the stability of a linear time-delay system for a given delay assuming the system to be unstable for zero delay. The main contribution of this paper is to consider a class of continuous first linear unstable processes with known parameters subject to time delays. As preliminary

\footnotetext{
${ }^{1}$ Electronics Department, University of Blida 1, Algeria

${ }^{2}$ Electronics Department, University of Blida 1, Algeria

${ }^{3}$ Electrical Engineering Department, ENP of Algiers, Algeria

* Abdelkader Maddi: Email: a_maddi@hotmail.com

Note: This paper has been presented at the 5th International Conference on Advanced Technology \& Sciences (ICAT'17) held in Istanbul (Turkey), May 09-12, 2017.
}

result, the standard case of system restricted to a smaller time delay than unstable time constant system is proved graphically by computing the first derivative and plots the graph of a function, the first derivative allows us to determine the critical points and several conditions of stability.

The remainder of this paper is organized as follows. We present the considered class of systems together with a recall of conditions stability lemmas in Section 2, which is followed by Walton and Marshall Method in Section 3. In Section 4, a truncation based methods is addressed. A graphical stabilization approach is presented in Section 5. A numerical example and simulations are presented in Section 6. Finally, concluding and remarks are given in Section 7.

\section{Preliminaries Results}

Let us consider the class of single input single output linear systems with time delay:

$$
\frac{Y(s)}{U(s)}=G(s) e^{-\tau s}=\frac{b}{s-a} e^{-\tau s}
$$

where $a>0$ and $b>0$ the parameters system, $\tau>0$ is the time delay, $G(s)$ is the delay free transfer function, $U(s)$ and $Y(s)$ are the input and output signals respectively.

In the following subsections, the existence conditions stability of closed-loop system is stated. Consider a proportional output feedback, as represented in Fig. 1, then, there exists a gain $K$ such that the closed-loop system is stable.

$$
\frac{Y(s)}{U(s)}=\frac{b e^{-\tau s}}{s-a+k b e^{-\tau s}}
$$

Lemma 1. Consider the delayed system (1) and the control scheme shown in Fig. 1, there exists a proportional gain $k$ such that the closed-loop system (2) is stable if and only if the time 
delay is small relatively to the time constant [7], it means $\tau a<1$.

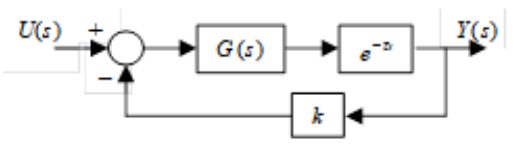

Fig. 1. Closed-loop system

\section{Walton and Marshall Method}

We recall a stability criterion for time delay systems with a single delay, which is due to Walton and Marshall in 1987. Time delay systems with a single delay have a characteristic equation of the following form:

$$
P(s, \tau)=N(s)+D(s) e^{-\tau s}
$$

where $N(s)$ and $D(s)$ are polynomials with real coefficients, such that degree $(D(s) \geq N(s))$ and verifying:

$$
\left\{\begin{array}{l}
N(j w)+D(j w) e^{-j w \tau}=0 \\
N(-j w)+D(-j w) e^{j w \tau}=0
\end{array}\right.
$$

The polynomial equation is given by,

$$
\psi\left(w^{2}\right)=N(j w) N(-j w)-D(j w) D(-j w)=0
$$

The solution of characteristic equation is,

$$
e^{j w_{i} \tau}=-\frac{N\left(-j w_{i}\right)}{D\left(-j w_{i}\right)}
$$

or

$$
e^{-j w_{i} \tau}=-\frac{N\left(j w_{i}\right)}{D\left(j w_{i}\right)}
$$

\subsection{Procedure}

The Walton-Marshall stability criterion consists in the following steps, as outlined below:

- Analyzing stability in the case free of delay, i.e. when $\tau=0$

- Increasing the time delay $\tau$ from zero to a strictly positive $\tau>0$, and evaluating how do the eigen-values move with increasing $\tau$

- Eliminating the exponentials to obtain a real polynomial $\psi\left(w^{2}\right)$

- Interesting to positive real solutions of the polynomial equation $\psi\left(w^{2}\right)=0$

- Noting that if $\psi\left(w^{2}\right)$ has no positive real roots, then there is no change in stability: if the system is stable (unstable) in the case free of delays $\tau=0$, then the system is stable (unstable) for all delay values $\tau>0$

- If this is not the case, we determine the positive roots of $\psi\left(w^{2}\right)=0$, and by using the characteristic equation: $N(j w)+D(j w) e^{-j w \tau}=0$, the time delay $\tau$ satisfies, if

$$
D(j w) \neq 0, e^{j w_{i} \tau}=-\frac{N\left(-j w_{i}\right)}{D\left(-j w_{i}\right)}
$$

\subsection{Example application}

Consider the characteristic equation of the closed-loop system (2), given by,

$$
P(s, \tau)=s-a+b k e^{-\tau s}=0
$$

where $a, b$ and $\tau$ are positives.

If $\tau=0$, then $s-a+b k=0$, Stability for, $b k>a$

For $\tau>0$,

$$
\begin{gathered}
\psi\left(w^{2}\right)=w^{2}+a^{2}-(b k)^{2} \\
w^{2}+a^{2}-(b k)^{2}=0 \\
w_{i}^{2}=(b k)^{2}-a^{2}
\end{gathered}
$$

For positive roots, $b k>a$, implies an asymptotic stability, if and only if, $\tau a<1$. But for negative roots, $0<b k<a$, there is no change in stability (unstable) in the case free of delays $\tau=0$.

The solution of characteristic equation is,

$$
e^{j w_{i} \tau}=\frac{b k}{a^{2}+w_{i}^{2}}\left(a+j w_{i}\right)
$$

where

$$
\left\{\begin{array}{l}
\cos \left(w_{i} \tau\right)=\frac{b k}{a^{2}+w_{i}^{2}} a=\frac{a}{b k} \\
\sin \left(w_{i} \tau\right)=\frac{b k}{a^{2}+w_{i}^{2}} w_{i}=\frac{w_{i}}{b k}
\end{array}\right.
$$

Or,

$$
\tan \left(w_{i} \tau\right)=\frac{w_{i}}{a}=\frac{w_{i} \tau}{a \tau}
$$

Finally, the asymptotical stability, if and only if, $0<a \tau<1$

\section{Truncation based methods}

The first order Pade approximation is given by,

$$
e^{-\tau s}=\frac{e^{-\tau s / 2}}{e^{+\tau s / 2}} \approx \frac{2-\tau s}{2+\tau s}
$$

We replace in the characteristic equation of the closed-loop system (2), given by (8), we obtain:

$$
P(s, \tau)=\tau s^{2}+(2-a \tau-b k \tau) s+(2 b k-2 a)=0
$$

Dressing the Routh-table, we obtained, 
Table 1. Routh-table

\begin{tabular}{lll}
\hline$s^{2}$ & $\tau$ & $(2 b k-2 a)$ \\
$s^{1}$ & $(2-a \tau-b k \tau)$ & 0 \\
$s^{0}$ & $(2 b k-2 a)$ & 0 \\
\hline
\end{tabular}

The number of roots of the polynomial that are in the right half plane is equal to the number of sign changes in the first column of Routh-array. And we show that all coefficients being positive are necessary for all roots to be located in the left half plane,

$$
\begin{aligned}
& \left\{\begin{array}{l}
\tau>0 \\
2-a \tau-b k \tau>0 \\
2 b k-2 a>0
\end{array}\right. \\
& \left\{\begin{array}{l}
a \tau<1 \\
b k>a
\end{array}\right.
\end{aligned}
$$

Since there is no sign change, the polynomial (16), has two poles in the left half plane.

\section{Graphical Stabilization Approach}

By computing the first derivative, it is a possible to plot the graph of a function with precision; the first derivative represents the slope of a function and allows us to determine its rate of change, the stationary and critical points allow us to obtain local minima, maxima and several conditions of stability.

\subsection{Methodology}

- Calculate the first derivative

- $\quad$ Find all stationary and critical points

- Create a table of variation by identifying the function at the stationary and critical points

- Whether the function is increasing or decreasing between the stationary and critical points

- Use the table to plot the graph

- Condition of asymptotic stability is given by the sign stationary point

\subsection{Example application}

Recall to (8), of the closed-loop system (2), given by,

$$
\begin{aligned}
& P(s, \tau)=s-a+b k e^{-\tau s}=0 \\
& b k=(a-s) e^{\tau s}
\end{aligned}
$$

We denote by $f$, the corresponding function,

$$
f(s, \tau)=b k=(a-s) e^{\tau s}
$$

And we plot the graph of the function after calculating the first derivative of $f(s)$,

$$
\frac{d f(s, \tau)}{d s}=[-1+(a-s) \tau] e^{\tau s}
$$

We obtain a stationary point when $f^{\prime}(s, \tau)=0$,

$$
\begin{aligned}
& {[-1+(a-s) \tau]=0} \\
& s=s_{0}=\frac{\tau a-1}{\tau}
\end{aligned}
$$

Create a table of variations by identifying the value of the function at the stationary point $f\left(s_{0}, \tau\right)=e^{\tau a-1} / \tau$.

Table 2. Table variations

\begin{tabular}{lcccc}
\hline$s$ & $-\infty$ & $s_{0}$ & $+\infty$ \\
$f^{\prime}(s, \tau)$ & & + & 0 & - \\
$f(s, \tau)$ & & & $f\left(s_{0}, \tau\right)$ &
\end{tabular}

There is thus one stationary point $S_{0}$ and no critical point since the derivative is well defined for all $S$. And we note that the value of $S_{0}$ can take any value positive (blue curve) or negative (green curve) as mentioned in Fig. 2.

For the blue curve, the condition of asymptotic stability is given by,

$$
a<b k<f\left(s_{0}, \tau\right), \quad \forall \tau a<1
$$

But for the green curve, there is no change in stability (unstable),

$$
0<b k<a, \quad \forall \tau>0
$$

In conclusion, we make the following condition, concerning the influence of the time delay $\tau$ in the stability analysis: $a<b k<f\left(s_{0}, \tau\right), \forall \tau a<1$ and there is no change in stability (unstable) for: $0<b k<a, \forall \tau>0$.

Finally, the result obtained by the proposed approach, is well justified and confirm the Walton-Marshall method.

Table 3. Example values for stability condition

$$
x=\tau a \quad \text { and } \quad 1<b k / a<f(x)
$$

$\begin{array}{ll}x & f(x) \\ 0.1 & 4.0657 \\ 0.2 & 2.2466 \\ 0.3 & 1.6553 \\ 0.4 & 1.3720 \\ 0.5 & 1.2131 \\ 0.6 & 1.1172 \\ 0.7 & 1.0583 \\ 0.8 & 1.0234 \\ 0.9 & 1.0054 \\ 1.0 & 1.0000\end{array}$




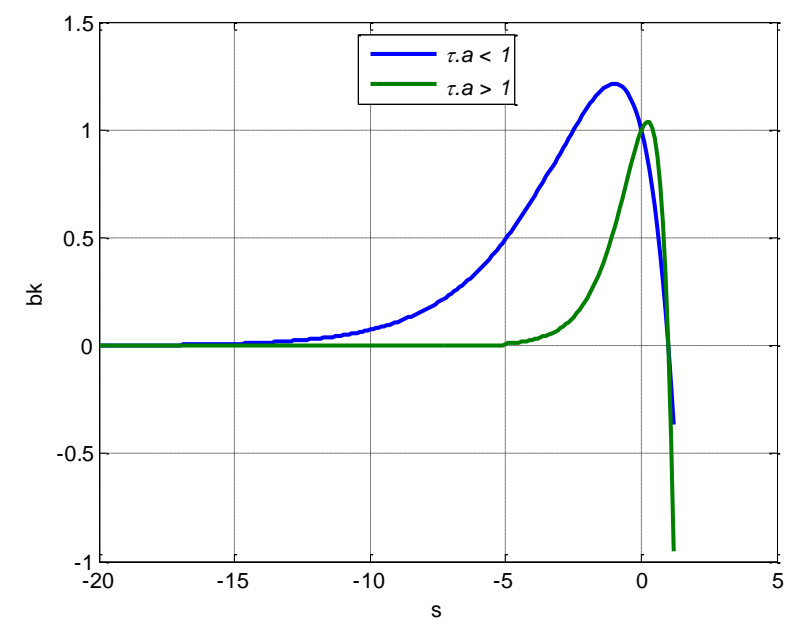

Fig. 2. Graph function

\section{Simulations and Results}

Let us consider a first order transfer function plus a dead time of plant model, which is given by,

$$
G(s)=\frac{b}{s-a} e^{-\tau s}
$$

where $a>0$ and $b>0$ the parameters system, $\tau \geq 0$ is the time delay.

Figure 3 shows the step response of open-loop system with $\tau=10$ where $b=1$ and $a=0.05$. Also, we can clearly see in Figures 4, 5 and 6, the step response of closed-loop system with different values of $\tau a<1$. Furthermore, it can be seen from comparison in Figure 7, for a small change of the parameter gain $k$ restricted to $a<b k<f\left(s_{0}\right)$ and $\tau a=0.5$. Figure 8 present a step response of closed-loop system when there is a small change in parameters system where $b=1$ and $a=0.05$.

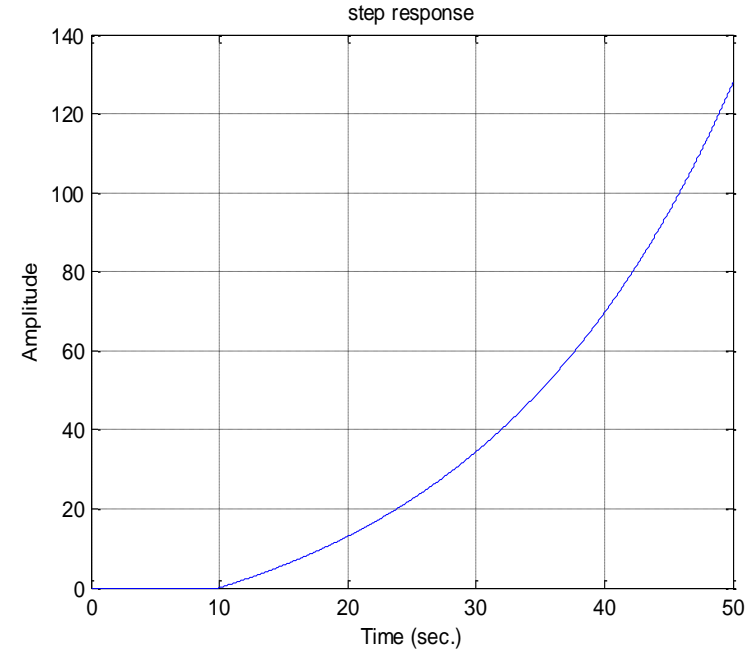

Fig. 3. Step response of open-loop system

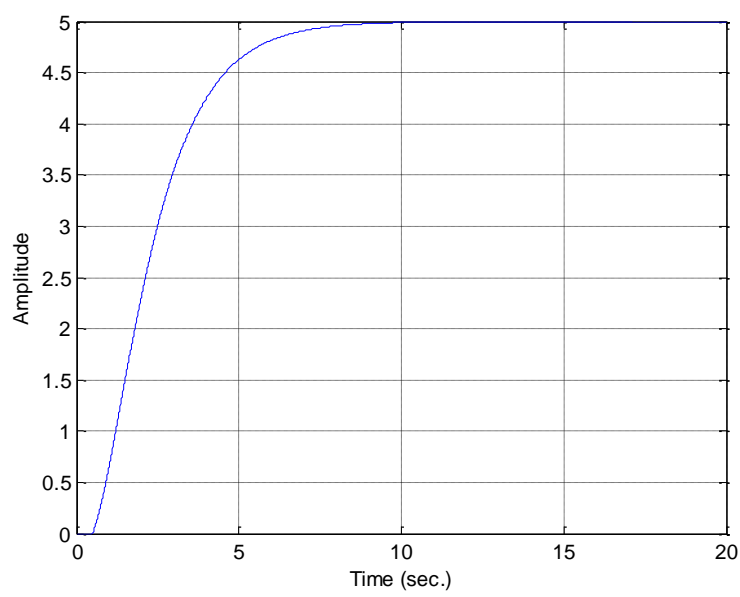

Fig. 4. Step response of cloused-loop system: $\tau a=0.5$ and $k=1.20$



Fig. 5. Step response of cloused-loop system: $\tau a=0.1$ and $k=2$

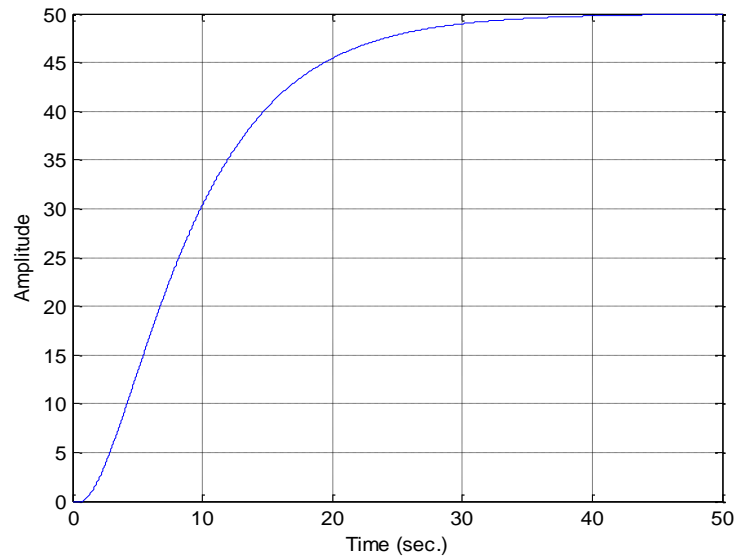

Fig. 6. Step response of closed-loop system: $\tau a=0.8$ and $k=1.02$ 


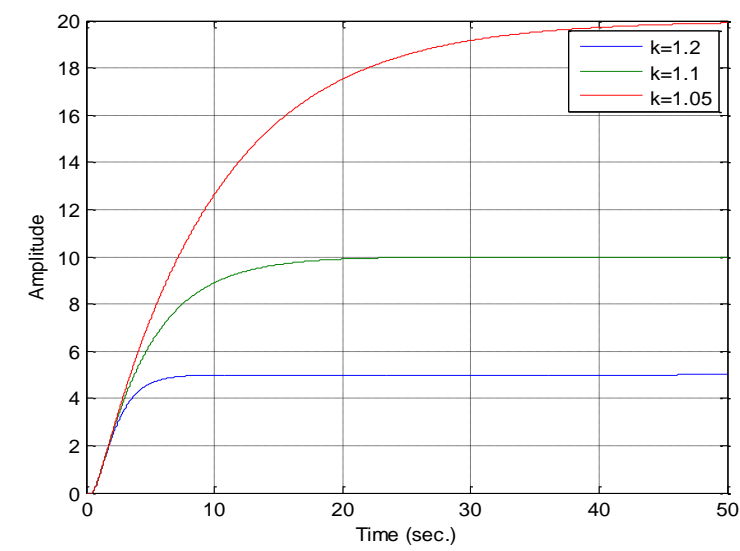

Fig. 7. Comparing step response of closed-loop system with $\tau a=0.5$

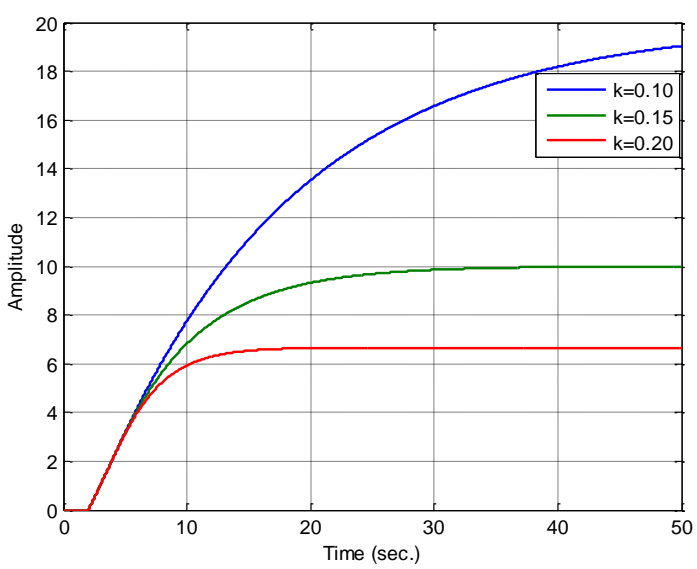

Fig. 8. Comparing step response of closed-loop system with $\tau a=0.1$

\section{Conclusion}

The analysis stability based on graphical stabilization approach is presented and analyzed for a class of unstable first order linear systems with time delay. Asymptotic stability is assured graphically by computing the first derivative and provided that the proportional gain such that the closed-loop system is stable if and only if the time delay is small relatively to the time constant. A numerical example for a first-order transfer function plus a dead time process has been studied and it has been showed by simulations that there exists a suitable choice of proportional gains $k$ such that the output signal converged to set point of the closed-loop system when $\tau a<1$, and the system is unstable in the case free of delays $\tau=0$, and becomes stable only for some strictly positive values of the delay.

Finally, the main advantage of the proposed approach is not only their simplicity, but also that it justifies and confirms the results obtained with Walton-Marshall method.

\section{References}

[1] S. Xu and T. Chen, "Robust Ho control for uncertain stochastic systems with state delay", IEEE Transactions on Automatic Control, 47(12): pp. 2089-2094, 2002.

[2] V. Kharitonov and A. Zhabko "Lyapunov-Krasovskii approach to the robust stability analysis of time delay systems", Automatica, 39: pp. 15-20, 2003.

[3] C. Hwang and JH. Hwang, "Stabilization of first order plus dead time unstable processes using PID controllers", IEEE Proc. Control Theory and Applications, 2004, 151(1): pp. 89-94.
[4] T. Liu, W. Zhang and D. Gu, "Analytical design of two degree of freedom control scheme for open loop unstable processes with time delay", Journal of Process Control, 2005, 5(15): pp. 559-572.

[5] J. E. Marshall, H. Gorecki, K. Walton and A. Korytowski, "Timedelay Systems, stability and performance criteria with applications", Ellis Horwood, 1992.

[6] G. Abdallah, P. Dorato, J. Benitez-Read, and R. Byrne, "Delayed positive feedback can stabilize oscillatory systems", $A C C$ '93, American Control Conference, pp. 3106-3107, 1993.

[7] B. Del-Muro-Cuellar, JF. Marquez-Rubio, M. Velasco-Villa and J. Alvarez-Ramirez, "On the Control of Unstable First Order Linear Systems with Large Time Lag: Observer Based Approach", European Journal of Control, 2012; 18(5): pp. 439-451.

[8] D. Peaucelle, D. Henrion and D. Arzelier, "Quadratic separation for feedback connection of an uncertain matrix and an implicit linear transformation", 16th IFAC World Congress, Prague, Czech Republic, July 2005.

[9] L. Orihuela, P. Millan, C. Vivas and F. Rubio, "Delay-dependent robust stability analysis for systems with interval delays", 2010 American Control Conference, Marriott Waterfront, Baltimore, MD, USA, June 30-July 02, 2010, pp. 4993-4998. 